\title{
EFECTOS DE LA SECUENCIACION DEL MATERIAL EN EL APRENDIZAJE DE LA LECTURA Y LA ESCRITURA
}

\author{
Blanca Patricia Ballesteros* \\ Rosa Agudelo de Rincón ${ }^{* *}$
}

Es innegable la importancia de las conductas de lectura y escritura ya que de ellas depende la adquisición de la mayoría de contenidos académicos. En consecuencia, es necesario garantizar su aprendizaje mediante métodos efectivos. Como se anotó en un trabajo anterior (Monografía Científica, Fascículo II, ICOLPE, 1975), a partir de la década del 50 se han utilizado los principios y técnicas de la Psicología del Aprendizaje en el campo específico de la lectura y escritura, con el objeto de determinar y controlar las variables implicadas en el proceso de adquisición, mantenimiento y generalización de estas conductas.

Respecto a las variables relevantes a las técnicas utilizadas en la programación de materiales de enseñanza, se han realizado diversos estudios en los cuales se ha verificado la efectividad de la igualación a la muestra y el desvanecimiento de los estímulos de apoyo en la adquisición de la conducta de lectura (Moore y Goldiamond, 1964; Gollin y Savoy, 1968; Díaz, 1974; Rayeck, 1974; Engeland, 1975). Igualmente, se ha comparado el desvanecimiento con la superposición encontrándose que es más efectivo el primero (Jeffrey y Jeffrey, 1972). Thimko (1974) comparó la igualación a la muestra sucesiva con la simultanea mostrando que fue más efectiva la simultánea. En cuanto a la utilización de estímulos de apoyo en los materiales de enseñanza, Knafle (1973) y Kiraly y Furlong (1974), verificaron la efectividad del color y de subrayado en la enseñanza de palabras, mientras que Galván y Ribes (1974) al utilizar dibujo y color en un material programado no encontraron diferencias significativas al compararlo con un programa tradicional.

Jeffrey (1958), Cadwell y Hall (1969) y Tawney (1972), encontraron que las secuencias instruccjonales elaboradas según un análisis sistemático de los elementos a enseñar, disminuyen el número de errores y el tiempo de aprendizaje. Jeffrey (1958) y Moore y Goldiamond (1964), mostraron que a través de una programación adecuada se logran discriminaciones complejas, por consiguiente, la topografía y complejidad fonética de las primeras letras que se enseñan deben ser fácilmente discriminables (Staiger, 1973). La programación del material debe incluir sólo un elemento nuevo cada vez para facilitar la discriminación al disminuir la probabilidad de error puesto que se ha demostrado que los errores conducen a más errores (Hively, 1962 y Terrace, 1966).

En relación con el material de escritura, Rayeck y Nesselroad (1972) y Wright y Allen (1975), sugieren que se utilicen técnicas de moldeamiento con desvanecimiento de estímulos de apoyo y que se tengan en cuenta las características topográficas de las letras. Por otra parte, Kirsch y Niedermayer (1973) encontraron que los ejercicios de copia fueron más efectivos que los de trazo desvanecido.

Respecto al sistema motivacional, Staats (1962 y 1964), Witlock y Bushell (1967), Heitzman (1970), Holt (1971), García, et al. (1974) y Agudelo y Torres (1974), han

\footnotetext{
* Psicóloga. Universidad Javeriana, Bogotá. Post-grado Investigación Curricular. Universidad Pedagógica Nacional.

** Psicóloga. Universidad Nacional de Colombia, Bogotá. Post-grado Investigación Curricular, Universidad Pedagógica Nacional. 
verificado la efectividad de reforzadores extrínsecos contingentes a la adquisición de las conductas de lectura y escritura. Lowe (1973) afirmó que la lectura se convierte en medio para adquirir otros contenidos reforzantes en sí mismos y que por tanto no es necesario reforzar explícitamente la adquisición de la conducta de lectura. En el trabajo ya mencionado (ICOLPE, 1975) se observó la limitación práctica de la aplicación en grupo de un sistema motivacional de puntos. Al suprimir éstos y aplicar sólo refuerzo social se comprobó su efectividad en la adquisición y mantenimiento de estas conductas.

Teniendo en cuenta que en la revisión anterior no se encuentran datos experimentales sobre los criterios que puedan seguirse para la elaboración de la secuencia de presentación de los elementos del material de lectura y escritura, y que en el estudio realizado en 1975 (Monografía Científica (II), ICOLPE), se vio la necesidad de mejorar la secuencia utilizada, se decidió comparar la efectividad de tres secuencias diferentes en el aprendizaje de lectura y escritura. Los criterios utilizados fueron: (1) Separación de rasgos topográfica y fonéticamente similares de acuerdo con los resultados de la investigación ya mencionada; (2) Agrupación gradual de acuerdo con la complejidad fonética de los sonidos; (3) Agrupación según los rasgos topográficos, con base en las recomendaciones de Rayeck y Nesselroad (1972) de enseñar agrupadas las letras con rasgos comunes.

\section{Variables}

La variable independiente fue la secuencia de aprendizaje establecida (secuencia 1: rasgos topográfica y fonéticamente similares separados; secuencia 2: rasgos fonéticos ordenados según su dificultad; secuencia 3: rasgos intermedios, ascendentes y descendentes. La variable dependiente fue el porcentaje de respuestas correctas en las pruebas de adquisición y mantenimiento de las conductas de lectura y escritura. Se entiende por adquisición, en lectura, la emisión de la conducta verbal correspondiente a un estímulo escrito y, en escritura, la conducta motora de escribir correspondiente a un estímulo verbal (escritura al dictado). Por mantenimiento se entiende las respuestas correctas en las pruebas periódicas de lectura y escritura posteriores a la adquisición. Se controlaron las variables relativas al material, procedimiento de aplicación, ambiente físico, horario de trabajo y sistema motivacional.

\section{Método}

Sujetos. 26 niños y niñas preescolares de la escuela Distrital Garcés Navas, de 5-8 años de edad, analfabetos, de clase media baja, y apareados según los resultados en la prueba de conductas precurrentes.

Ambiente físico. Salón de clase de la concentración escolar Garcés Navas de Bogotá. lluminación, ventilación y pupitres adecuados.

Instrumentos. Material programado de lectura y escritura de las 42 palabras utilizadas; hojas de registro de la ejecución de los sujetos en cada paso del material y de los resultados de las pruebas; pruebas de conductas precurrentes, de adquisición y de mantenimiento.

Procedimiento. Se aplicó la prueba de lectura y escritura del material a 50 niños y niñas y se seleccionaron 40 sujetos analfabetas a quienes se les aplicó la prueba de conductas precurrentes. Con base en estos resultados se seleccionaron y aparearon 30 sujetos, conformando los tres grupos experimentales: Grupo 1-Secuencia 1, Grupo 2Secuencia 2, Grupo 3-Secuencia 3. 
Los grupos trabajaron en el mismo salón pero con un experimentador cada uno. Después de un entrenamiento en el manejo del material se procedió a la aplicación de éste. En primer lugar se enseñaron las vocales y cuando en cada grupo se probó su adquisición, se comenzó la aplicación de las palabras programadas, en su orden. La enseñanza de cada elemento incluía primero la lectura y luego la escritura (moldeamiento, imitación y control verbal). Diariamente se realizaron las pruebas de adquisición y mantenimiento, las cuales eran individuales en lectura y colectivas en escritura, controlando la oportunidad de copia; como sistema motivacional se aplicó refuerzo social (expresiones como "muy bien", "correcto", "cómo lees de lindo", etc.) en forma intermitente a cada sujeto.

Cuando los sujetos emitían respuestas incorrectas en las pruebas de adquisición o mantenimiento, se hacían planas controladas por el experimentador.

\section{Resultados}

En cuanto al análisis del material de lectura, se encontró que los ensayos correspondientes a discriminación sin muestra presentaron mayor porcentaje de error en los 3 grupos. En el material de escritura no se encontró consistencia en los errores a través de las tres secuencias y en las últimas palabras el grupo 2 presentó más errores. Solamente en 8 de las 42 palabras se cometieron errores en el paso de escritura al dictado, aunque no coincidieron los 3 grupos en la misma palabra.

Las confusiones más frecuentes, entre letras, fueron las siguientes: d-b; n-c; f-t; j-i; ñ -n; p-q; o-a.

Tabla 1

Palabras que obtuvieron menos del $75 \%$ de adquisición (\% correspondiente a dos sujetos)

\begin{tabular}{|c|c|c|c|c|c|c|c|c|c|c|c|c|c|}
\hline Palabras & $\begin{array}{c}\text { Sec. } \\
L\end{array}$ & $\frac{\text { I }}{E}$ & $\begin{array}{c}\text { Sec. } \\
\text { L }\end{array}$ & $\begin{array}{l}\text { II } \\
\text { E }\end{array}$ & $\begin{array}{c}\text { Sec. } \\
\text { L }\end{array}$ & $\begin{array}{l}\text { III } \\
\text { E }\end{array}$ & Palabras & $\begin{array}{c}\text { Sec. } \\
\text { L }\end{array}$ & $\begin{array}{l}\text { I } \\
E\end{array}$ & $\begin{array}{c}\text { Sec. } \\
L\end{array}$ & $\begin{array}{l}\text { II } \\
\text { E }\end{array}$ & $\begin{array}{c}\text { Sec. } \\
L\end{array}$ & $\begin{array}{c}\text { III } \\
\text { E }\end{array}$ \\
\hline $\begin{array}{l}\text { mamá } \\
\text { papá } \\
\text { loma } \\
\text { nene }\end{array}$ & $\begin{array}{l}x \\
x\end{array}$ & & $X$ & $x$ & & $x$ & $\begin{array}{c}\text { quema } \\
\text { loro } \\
\text { kilo } \\
\text { cine }\end{array}$ & $\begin{array}{l}x \\
x\end{array}$ & & $\begin{array}{l}x \\
x\end{array}$ & $\begin{array}{l}x \\
x \\
x\end{array}$ & $x$ & \\
\hline saco & $x$ & & & & & & cigüeña & & & $x$ & & $x$ & $x$ \\
\hline $\begin{array}{c}\text { boca } \\
\text { rosa }\end{array}$ & $x$ & & $x$ & $x$ & $X$ & $\begin{array}{l}x \\
x\end{array}$ & $\begin{array}{c}\text { clavo } \\
\text { tablero }\end{array}$ & $\begin{array}{l}x \\
X\end{array}$ & & & & $x$ & \\
\hline $\begin{array}{c}\text { carro } \\
\text { tina } \\
\text { gato } \\
\text { querra }\end{array}$ & $\begin{array}{l}x \\
x\end{array}$ & & $x$ & $x$ & & & $\begin{array}{l}\text { plato } \\
\text { globo } \\
\text { atleta } \\
\text { crema }\end{array}$ & & & $X$ & & $\begin{array}{l}x \\
x\end{array}$ & $x$ \\
\hline fila & $X$ & & & & $x$ & $x$ & bruja & & & $x$ & & $X$ & \\
\hline $\begin{array}{l}\text { vaca } \\
\text { dedo } \\
\text { gitana } \\
\text { chino }\end{array}$ & $x$ & & $\begin{array}{l}x \\
x\end{array}$ & $x$ & $\begin{array}{l}x \\
x \\
x\end{array}$ & & $\begin{array}{l}\text { grano } \\
\text { fruta } \\
\text { madre } \\
\text { padre }\end{array}$ & $X$ & & $X$ & & $\begin{array}{l}x \\
x \\
x\end{array}$ & \\
\hline
\end{tabular}

Respecto a la variable adquisición se observó menor adquisición de lectura respecto a 
escritura en los 3 grupos, aunque no coinciden en las mismas palabras. En las combinaciones (tl, pl, tr, gl, etc.) el grupo 1 presentó menor adquisición (tabla 1).

Para analizar los datos estadísticamente se aplicaron coeficientes de regresión de modelo lineal. En lectura se utilizaron las variables orden de la palabra en la secuencia y número de errores cometidos en el material, y en escritura, orden de la palabra y puntaje de adquisición en lectura. En la tabla 2 se presentan los resultados principales del análisis de regresión para cada palabra. En la segunda columna se presentan las palabras para las cuales se ajusta el modelo lineal de regresión. Se consideró un nivel máximo de significación de 0.10 debido a la naturaleza de la escala de medición de las variables. En los casos donde el modelo no alcanzó este nivel de significación, se presentan en la última columna los coeficientes de correlación correspondiente. En la tercera columna $(R$ cuadrado) se presenta el coeficiente de determinación: proporción de variación en la variable dependiente (lectura o escritura) explicada por su relación con las variables independientes consideradas simultáneamente.

La cuarta columna corresponde a las variables independientes en función de las cuales está la adquisición. La x representa el factor que tuvo mayor peso en la ecuación y a su vez, si éste fue o no significativo.

Los principales datos obtenidos fueron:

a) El modelo lineal resultó significativo en un $76 \%$ de los casos (aproximadamente 32 palabras) para adquisición de lectura. Sin embargo, para adquisición de escritura sólo resultó significativo en $54 \%$ de los casos.

b) En términos de variación explicada (coeficiente de determinación), en algunos casos, a pesar de la significatividad del modelo se obtuvo un coeficiente bajo. Este hecho se podría explicar por la influencia de otros factores como inasistencia de los sujetos en distintas ocasiones, diferencias individuales de los experimentadores o la relación misma de las variables consideradas en el modelo con otras no presentes en éste.

En escritura, a pesar del menor número de modelos significativos los coeficientes de determinación son mayores.

c) Si se analiza de acuerdo con lo esperado, el orden, en su relación con lectura, sólo resultó significativo en 4 palabras: en 23 palabras no fue significativo. En escritura, el orden cumplió con la relación esperada en el $84 \%$ de los casos, aunque no de forma significativa. 
Resultados principales del análisis de regresión aplicado a los puntajes de lectura y escritura para cada palabra $(=0.10)$

\begin{tabular}{|c|c|c|c|c|c|c|c|c|c|}
\hline \multicolumn{4}{|c|}{$F$} & \multicolumn{4}{|c|}{ Variables Independientes } & \multirow{2}{*}{\multicolumn{2}{|c|}{$\begin{array}{c}\text { Correlación } \\
\text { Parcial }\end{array}$}} \\
\hline \multicolumn{3}{|c|}{ Modelo Lineal } & $\mathbf{R}$ & & den & No & irrores & & \\
\hline Palabras & Sig. & No.sig & Cuadrado & Sig. & No.sig & Sig. & No.sig. & $\mathrm{Yx} 1$ & $\mathrm{Yx} 2$ \\
\hline mamá & $x$ & & .48 & $-\mathrm{X}$ & & & & & \\
\hline papá & $x$ & & .30 & $-\mathrm{X}$ & & & & & \\
\hline coco & $x$ & & .25 & & & $x$ & & & \\
\hline loma & $x$ & & .30 & & & & $-\mathrm{X}$ & & \\
\hline nene & & $x$ & .005 & & & & & -0.003 & 0.064 \\
\hline saco & $x$ & & .50 & $-\mathrm{X}$ & & & & & \\
\hline boca & $x$ & & .22 & & & $-\mathrm{X}$ & & & \\
\hline rosa & & $x$ & .090 & & & & & 0.27 & -0.19 \\
\hline carro & $x$ & & .45 & & & $-x$ & & & \\
\hline tina & $x$ & & .34 & $-x$ & & & & & \\
\hline uña & & $x$ & .10 & & & & & 0.24 & -0.28 \\
\hline gato & & $x$ & .045 & & & & & -0.086 & -0.20 \\
\hline guerra & $x$ & & .44 & & & $-x$ & & & \\
\hline & & $x$ & .12 & & & & & -0.33 & 0.22 \\
\hline dedo & $x$ & & .33 & $-x$ & & & & & \\
\hline vaca & $x$ & & .71 & & & $-x$ & & & \\
\hline llave & $x$ & & .68 & & & $-x$ & & & \\
\hline ojo & $x$ & & .44 & & & $-x$ & & & \\
\hline zapato & $x$ & & .42 & & & $-x$ & & & \\
\hline hilo & & $X$ & .17 & & & & & -0.15 & $-0.41^{*}$ \\
\hline gitana & $x$ & & .27 & & & $-x$ & & & \\
\hline taxi & $x$ & & .27 & & & $-x$ & & & \\
\hline
\end{tabular}

TABLA 2 (cont.) 


\begin{tabular}{|c|c|c|c|c|c|c|c|c|c|}
\hline \multicolumn{4}{|c|}{$\mathbf{F}$} & \multicolumn{4}{|c|}{ Variables Independientes } & \multirow{2}{*}{\multicolumn{2}{|c|}{$\begin{array}{c}\text { Correlación } \\
\text { Parcial }\end{array}$}} \\
\hline \multicolumn{3}{|c|}{ Modelo Lineal } & \multirow{2}{*}{$\begin{array}{c}\mathbf{R} \\
\text { Cuadrado }\end{array}$} & \multicolumn{2}{|c|}{ Orden } & \multicolumn{2}{|c|}{ Adquis. Lect. } & & \\
\hline Palabras & Sig. & No.sig & & Sig. & No.sig & Sig. & No.sig. & Yx1 & $Y \times 2$ \\
\hline chino & & $\mathrm{X}$ & 0.040 & & & & & -0.11 & 0.17 \\
\hline quema & $x$ & & 0.58 & & & $\mathrm{x}$ & & & \\
\hline Ioro & $x$ & & 0.68 & & & $x$ & & & \\
\hline yuca & $x$ & & 0.10 & & & & & & \\
\hline kilo & $x$ & & 0.87 & & & $x$ & & & \\
\hline cine & & $x$ & 0.09 & & & & & 0.24 & 0.24 \\
\hline cigüeña & & $x$ & 0.057 & & & & & 0.24 & -0.065 \\
\hline clavo & & $x$ & 0.093 & & & & & -0.30 & -0.20 \\
\hline tablero & & $x$ & 0.006 & & & & & -0.039 & -0.076 \\
\hline & $x$ & & 0.0 & & & & & & \\
\hline plato & $x$ & & 0.0 & & & & & & \\
\hline globo & & $x$ & 0.009 & & & & & -0.23 & -0.28 \\
\hline atleta & $x$ & & 0.37 & & & $\mathrm{x}$ & & & \\
\hline crema & $x$ & & 0.0 & & & & & & \\
\hline bruja & $x$ & & 0.37 & & & $x$ & & & \\
\hline fruta & $x$ & & 0.0 & & & & & & \\
\hline grano & $x$ & & 0.0 & & & & & & \\
\hline madre & $x$ & & .32 & & & $x$ & & & \\
\hline prado & $x$ & & 0.0 & & & & & & \\
\hline tren & $x$ & & 0.0 & & & & & & \\
\hline
\end{tabular}

TABLA 2 (cont.) 


\begin{tabular}{|c|c|c|c|c|c|c|c|c|c|}
\hline \multicolumn{4}{|c|}{$F$} & \multicolumn{4}{|c|}{ Variables Independientes } & \multirow{2}{*}{\multicolumn{2}{|c|}{$\begin{array}{c}\text { Correlación } \\
\text { Parcial }\end{array}$}} \\
\hline \multicolumn{3}{|c|}{ Modelo Lineal } & $\mathbf{R}$ & & den & & rores & & \\
\hline Palabras & Sig. & No.sig & Cuadrado & Sig. & No.sig & Sig & No.sig. & $\mathrm{Y} \times 1$ & $\mathrm{Yx} 2$ \\
\hline chino & & $x$ & .074 & & & & & -0.12 & -0.17 \\
\hline quema & $\mathrm{X}$ & & .30 & & & $-x$ & & & $-0.40^{*}$ \\
\hline loro & & $x$ & .17 & & & & & -0.14 & \\
\hline yuca & $x$ & & .41 & & & $-\mathrm{X}$ & & & \\
\hline kilo & $x$ & & .45 & $\mathrm{X}$ & & & & & \\
\hline cine & & $x$ & .17 & & & & & 0.32 & -0.35 \\
\hline cigüeña & $x$ & & .54 & & & $-\mathrm{X}$ & & & \\
\hline clavo & $x$ & & .52 & & & $-X$ & & & \\
\hline tablero & $x$ & & .25 & & & $-X$ & & & \\
\hline flor & $x$ & & .45 & & & $-x$ & & & \\
\hline plato & $x$ & & .48 & & & $-\mathrm{X}$ & & & \\
\hline globo & $x$ & & .52 & & & $-X$ & & & \\
\hline atleta & $x$ & & .31 & & & $-X$ & & & \\
\hline crema & $x$ & & .20 & $x$ & & $-x$ & & & \\
\hline bruja & $x$ & & .52 & & & $-x$ & & & \\
\hline fruta & $x$ & & .33 & & & $-x$ & & & \\
\hline grano & $x$ & & .35 & & & $-x$ & & & \\
\hline madre & & $\mathrm{X}$ & .17 & & & & & -0.24 & $-0.40^{*}$ \\
\hline prado & $x$ & & .23 & $x$ & & & & & \\
\hline tren & $x$ & & .24 & & & $-x$ & & & \\
\hline
\end{tabular}


TABLA 2 (cont.)

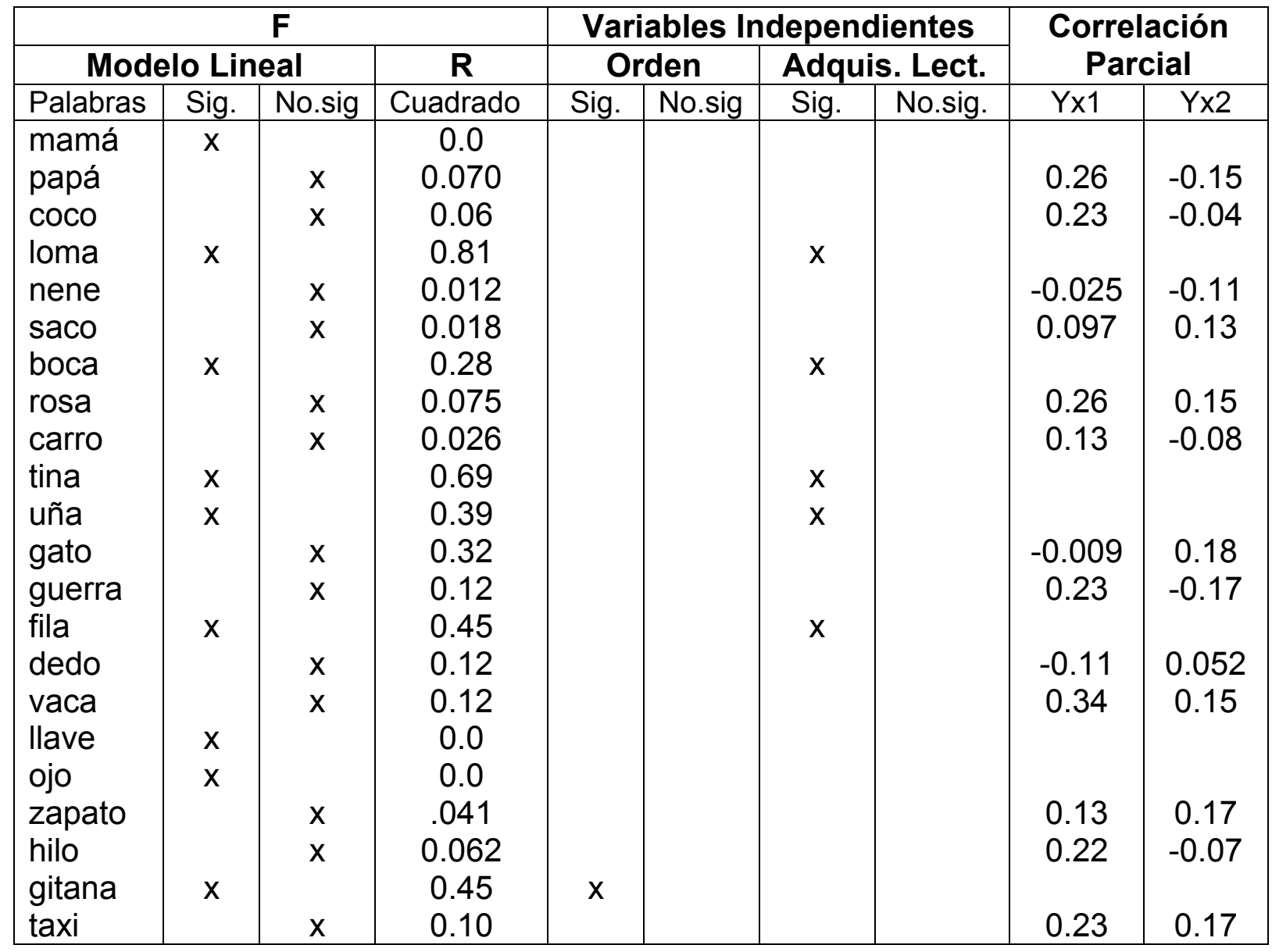

En cuanto al número de errores, su peso resultó significativamente mayor que el orden en el $54 \%$ de los casos. En escritura, el factor de mayor peso fue la adquisición de lectura, el cual fue significativo en el $26 \%$ de los casos.

En cuanto a la variable mantenimiento, se encontró que la conducta de lectura tuvo mayor mantenimiento en cuanto a número de respuestas incorrectas en las pruebas de cada palabra. En escritura, en la secuencia 2 no se obtuvieron mas de 3 respuestas incorrectas en ninguna palabra. La secuencia 3 fue la que menor mantenimiento tuvo en la mayoría de las palabras. Para analizar si las diferencias entre los 3 grupos eran significativas, se aplicó la prueba de Duncan para cada palabra, a un nivel de significación de 0.05 . En la tabla 3 se presentan las palabras en las cuales la prueba fue negativa para los 3 grupos.

Como puede observarse, cerca del $43 \%$ de las palabras presentaron Duncan no significativo tanto en lectura como en escritura, aun teniendo diferencias de orden de 10 , 14,18 y 21 unidades de por medio. El $25 \%$ de las palabras que obtuvieron Duncan significativo coinciden en lectura y escritura. De las 13 palabras correspondientes a las combinaciones, 9 presentaron diferencias significativas, la mayoría de las cuales están en el grupo 2 respecto a los grupos 1 y 3 . En la tabla 4 se presentan con una $x$ las palabras que en cada secuencia mostraron diferencias significativas respecto a las otras. Puede concluirse que la secuencia 1 obtuvo el menor promedio de mantenimiento en mayor número de palabras y a su vez difirió de la secuencia de mayor promedio, la cual para algunas palabras fue la 2 y para otras la 3 . 
Tabla 3

Palabras en las cuales la prueba Duncan fue negativa

\begin{tabular}{|c|c|c|}
\hline \multicolumn{2}{|r|}{ Lectura } & \multirow{2}{*}{$\begin{array}{l}\text { Escritura } \\
\text { loma } \\
\end{array}$} \\
\hline${ }^{*}$ mama & Hilo & \\
\hline "papá & ${ }^{*} \operatorname{taxi}$ & rosa \\
\hline${ }^{*} \mathrm{COCO}$ & Chino & fila \\
\hline nene & "yuca & dedo \\
\hline saco & ${ }^{*}$ kilo & Ilave \\
\hline *boca & ${ }^{*}$ cine & zapato \\
\hline${ }^{*}$ carro & ${ }^{*}$ clavo & tablero \\
\hline${ }^{*}$ tina & ${ }^{*}$ flor & madre \\
\hline *uña & ${ }^{*}$ plato & tren \\
\hline *gato & Globo & \\
\hline *guerra & Atleta & \\
\hline *vaca & *grano & \\
\hline "ojo & & \\
\hline
\end{tabular}

\section{Discusión}

Los errores cometidos en el material de lectura pueden indicar la necesidad de incluir pasos adicionales entre los pasos con muestra y las de sólo reconocimiento, pues en éstas últimas se utilizaron estímulos distractores muy similares topográficamente.

Al comparar los datos de adquisición con los de mantenimiento se encontró consistencia respecto a la lectura: los grupos con menor adquisición obtuvieron menor mantenimiento, lo cual permitiría ampliar los resultados del análisis de regresión en el sentido de esperar una relación directa entre adquisición y mantenimiento, siempre y cuando se den las condiciones para garantizar la práctica de lo adquirido. Respecto a la conducta de escritura, se corroboran los hallazgos de un estudio anterior (ICOLPE, 1975) que llevarían a afirmar la mayor complejidad de esta conducta.

Aunque la variable independiente del estudio fue el orden de las palabras en la secuencia, en los análisis de regresión este factor no resultó significativamente determinante en la mayoría de los casos. Esto puede explicarse, por una parte, porque para cada secuencia los criterios de programación se basaron en los principios básicos del aprendizaje y sólo en algunas palabras hubo diferencias de posición entre 8 y 20 unidades; por otra parte, porque resulta imposible controlar el número o el tipo de error y esto va en detrimento del aprendizaje, como se ha demostrado experimentalmente y se corrobora en este estudio.

Por consiguiente se puede afirmar que para la programación de una secuencia de aprendizaje de lectura y escritura, lo más importante de controlar es la ordenación en función de la dificultad fonética y topográfica de los elementos y la probabilidad de error en cada paso del material.

A pesar de que en la Tabla 1, aparentemente la escritura es independiente de la lectura, de acuerdo con el análisis de regresión sí se puede concluir que la previa adquisición de

\footnotetext{
* Duncan negativo en lectura y escritura 
la lectura facilitó la adquisición de escritura (Tabla 2) en los casos en los cuales el modelo lineal resulté significativo.

Respecto a la medición de las variables dependientes, la de adquisición de lectura presenta dificultades en dos sentidos: si se mide inmediatamente después de la terminación del material, se corre el riesgo de no evaluar la respuesta esperada sino una respuesta más específica ante una situación de estímulos determinada (lo que para algunos autores sería memoria inmediata), por otra parte, si se mide al terminar la escritura 0 al otro día, existen factores contaminantes, tales como práctica adicional. La medición de adquisición de escritura también presenta las mismas dificultades, aunque implica una conducta motora diferente, probablemente menos sensible a la inmediatez. La variable adquisición se tomó como una variable dicotómica debido a la definición misma de esta variable, ya que sólo permite que se lea o se escriba la palabra en su totalidad.

Tabla 4

Palabras que en cada secuencia obtuvieron promedios bajos que difirieron de las demás

\begin{tabular}{|c|c|c|c|c|c|c|}
\hline & \multicolumn{2}{|c|}{ Sec. I } & \multicolumn{2}{|c|}{ Sec. II } & \multicolumn{2}{|c|}{ Sec. III } \\
\hline Palabras & $\mathbf{L}$ & $E$ & $\mathbf{L}$ & $E$ & $\mathbf{L}$ & $E$ \\
\hline loma & & & & & & \\
\hline nene & & & & & & \\
\hline saco & & & & & & \\
\hline rosa & $x$ & & & & & \\
\hline guerra & & $x$ & & & & \\
\hline fila & $x$ & & & & & \\
\hline dedo & $x$ & & & & & \\
\hline llave & $x$ & & & & & \\
\hline zapato & $x$ & & & & & \\
\hline hilo & & $x$ & & & & \\
\hline gitana & $x$ & $x$ & & & & \\
\hline chino & & $x$ & & & & \\
\hline quema & $x$ & & & & & \\
\hline $\begin{array}{c}\text { loro } \\
\text { ciqüeña }\end{array}$ & $x$ & $x$ & $x$ & $\mathrm{x}$ & & \\
\hline tablero & $x$ & ר & & & & \\
\hline globo & & & & & & \\
\hline $\begin{array}{l}\text { atleta } \\
\text { bruja }\end{array}$ & & $\mathrm{X}$ & & & & $x$ \\
\hline fruta & $\mathrm{x}$ & $x$ & & & $\mathrm{x}$ & \\
\hline madre & $\mathrm{x}$ & & & & & \\
\hline $\begin{array}{c}\text { prado } \\
\text { tren }\end{array}$ & $x$ & $x$ & $\mathrm{x}$ & & $x$ & $x$ \\
\hline crema & $\mathrm{x}$ & & & & & \\
\hline
\end{tabular}

Uno de los aspectos importantes de este estudio se encuentra a nivel del sistema motivacional utilizado. Solamente se aplicó refuerzo social intermitente y se observó que éste fue suficiente para la adquisición y el mantenimiento de las conductas. Este hecho permite mayor funcionalidad del método en el salón de clase, siempre y cuando el refuerzo se aplique de forma consistente y no vaya acompañado de estimulación aversiva.

Con base en los puntos anteriores conviene hacer una serie de recomendaciones:

1. Programar en los primeros lugares de la secuencia las palabras topográfica y 
fonéticamente más simples.

2. Aumentar los pasos de reconocimiento (discriminación sin muestra) y asegurar la emisión verbal ante el estímulo correcto, en el material de lectura.

3. Asegurar una adquisición sin errores con el fin de evitar pasos correctivos y garantizar el mantenimiento.

4. Utilizar el dibujo y la distribución horizontal de los estímulos.

5. En ausencia de otro tipo de reforzadores, aplicar en forma sistemática el refuerzo social (expresiones verbales y físicas de aprobación). 
AGUDELO, R. y TORRES, M. Método programado para enseñanza individual de lectura y escritura. Centro de Investigaciones de la Universidad Pedagógica Nacional. Bogotá, 1974 (sin publicar).

CADWELy HALL. The influence of concept training on letter discrimination. Child Development. 1969, 40: 63-71.

DIAZ, Jorge E. Transferencia del control de estímulos en una ejecución de lectura; en Aportaciones al Análisis de la Conducta. México: Ed. Trillas, 1974.

ENGELAND, Byron. Effects of errorless training on teaching children to discriminate letters. Jour of Applied Psychology, 1975. Vol. 60 (40): 533536.

GALVAN, ME. y RIBES, E. Evaluación de diferentes procedimientos para establecer conducta textual en niños preescolares; en Aportaciones al Análisis de la Conducta. México: Ed. Trillas, 1974.

GARCIA, CH. et al. Efectos diferenciales de varias contingencias sobre la exactitud en un programa de escritura; en Aportaciones al Análisis de la Conducta. México: Ed. Trillas, 1974.

GOLLIN, E. y SAVOY, F. Fading procedures and conditional discrimination in children. Jour of Experimental Analysis of Behavior, 1968; ii: 443-451.
HOLT, Gary. Effect of reinforcement contingencies on increasing programmed reading and mathematics behavior in first grade children. Jour of Experimental Child Psychology, 1971.12: 362-369

ICOLPE. Método de Alfabetización. Monografía Científica, Fascículo II, Bogotá, 1975.

JEFFREY, W.E. Variables in early discrimination learning. Child Development, 1958, 29: 269-275.

EFFREY, W.E. y JEFFREY, Sh. Effects of fading procedures in the acquisition of oral reading. Jour of Applied Behavior Analysis. 1972, Vol. 5.

KIRALY, John y URLONG, Alexandra. Teaching words to kindergarten children with picture configuration and initial sound cues in prometing procedures. Jour of Educational Research, 1974 (Oct.) Vol. 67 (7): 295-298.

KIRSCH, E. y NIEDERMAYER, F. The effects of tracing prompts and discrimination training on kindergarten handwriting performance. Jour of Educational Research, 1973. (Oct.) Vol. 67 (2): 81-86.

KNAFLE, J. Color, underlining and word shape cues in word structure detection. Reading Research Quarterly. 1973, Vol. 8 (4): 502-523. 
HEITZMAN, Andrew. Effects of a Token reinforcement system on reading and arithmetic, skills learning of migrant primary school pupils. Jour of Educational Research, 1970, Vol. 63 (10).

HIVELY, W. Programming stimuli in matching to sample. Jour of experimental analysis of behavior. 1962, 5: 279-298.

RAYECK, Eli. y NESSELROAD, Elizabeth. Behavior Principles in teaching spelling and composition: en Behavior Analysis and Education, Editado por George Semb, Kansas, 1972

STAATS, A.W. Et. al. The conditioning of textual responses using extrinsec reinforcers. Jour of Experimental Analysis of Behavior. 1964,7:149.

STAIGER, Ralph (ed). The teaching of reading. Suiza; UNESCO/Gimn, 1973.

TAWNEY, James. Training letters discrimination in four years old children. Jour of Applied Behavior Analysis. 1972, 5: 455-467
MOORE y GOLDIAMOND. Errorless establishment of visual discrimination using fading procedures. Jour of Experimental Analysis of Behavior. 1974, 7: 272.

RAYECK, Eli. Relación funcional entre una secuencia de atención y un programa elemental de lectura; en Aportaciones al Análisis de la Conducta. México: Ed. Trillas, 1974.

TERRACE, H .S. Discrimination training with and without errors. Jour of Experimental Analysis of Behavior. 1963, 6:1-27.
THIMKO, Henry. Effects of discrimination training made and letter similarity on paired associate learning. Jour of Educational Research, 1974, Vol. 68, Octubre (2): 71-72.

WITLOCK, C. y BUSHELL, D. (Jr.). Some effects of "back up" reinforcers on reading behavior. Jour of Experimental Child Psychology. 1967 5:50-57

WRIGHT, Jane y ALLEN, Elizabeth. Ready to write. Elementary School Journal, 1975. Vol. 75 (7): 430-435. 